\title{
ON AN ASYMPTOTICALLY LINEAR ELLIPTIC DIRICHLET PROBLEM
}

\author{
ZHITAO ZHANG, SHUJIE LI, SHIBO LIU, AND WEIJIE FENG
}

Received 2 July 2002

Under very simple conditions, we prove the existence of one positive and one negative solution of an asymptotically linear elliptic boundary value problem. Even for the resonant case at infinity, we do not need to assume any more conditions to ensure the boundness of the (PS) sequence of the corresponding functional. Moreover, the proof is very simple.

\section{Introduction}

In this paper, we consider the existence of one-signed solutions for the following Dirichlet problem:

$$
\begin{aligned}
-\Delta u & =f(x, u), \quad x \in \Omega, \\
u & =0, \quad x \in \partial \Omega,
\end{aligned}
$$

where $\Omega$ is a bounded domain in $\mathbb{R}^{N}(N \geq 1)$ with smooth boundary $\partial \Omega$. The conditions imposed on $f(x, t)$ are as follows:

$\left(\mathrm{f}_{1}\right) f \in C(\Omega \times \mathbb{R}, \mathbb{R}) ; f(x, 0)=0$, for all $x \in \Omega$.

$\left(\mathrm{f}_{2}\right) \lim _{|t| \rightarrow 0}(f(x, t) / t)=\mu, \lim _{|t| \rightarrow \infty}(f(x, t) / t)=\ell$ uniformly in $x \in \Omega$.

Since we assume $\left(\mathrm{f}_{2}\right)$, problem $(1.1)$ is called asymptotically linear at both zero and infinity. This kind of problems have captured great interest since the pioneer work of [1]. For more information, see $[2,3,4,5,6,7,8,11,12]$ and the references therein.

Obviously, the constant function $u=0$ is a trivial solution of problem (1.1). Therefore, we are interested in finding nontrivial solutions. Let $F(x, u)=$ $\int_{0}^{u} f(x, s) d s$. It follows from $\left(\mathrm{f}_{1}\right)$ and $\left(\mathrm{f}_{2}\right)$ that the functional

$$
J(u)=\frac{1}{2} \int_{\Omega}|\nabla u|^{2} d x-\int_{\Omega} F(x, u) d x
$$


is of class $C^{1}$ on the Sobolev space $H_{0}^{1}:=H_{0}^{1}(\Omega)$ with norm

$$
\|u\|:=\left(\int_{\Omega}|\nabla u|^{2}\right)^{1 / 2}
$$

and the critical points of $J$ are solutions of (1.1). Thus we will try to find critical points of $J$. In doing so, we have to prove that the functional $J$ satisfies the (PS) condition.

We denote by $0<\lambda_{1}<\lambda_{2} \leq \lambda_{3} \leq \cdots \leq \lambda_{i} \leq \cdots$ the eigenvalues of $\left(-\Delta, H_{0}^{1}\right)$ with eigenfunctions $\phi_{i}$. If $\ell$ is an eigenvalue of $\left(-\Delta, H_{0}^{1}(\Omega)\right)$, then the problem is resonant at infinity. This case is more delicate. To ensure that $J$ satisfies the (PS) condition usually one needs to assume additional conditions, such as the wellknown Landesman-Lazer condition, see, for example, $[3,4]$; the angle condition at infinity, see [2].

Recently, in the case $0 \leq \mu<\lambda_{1}<\ell$, Zhou [12] obtained a positive solution of problem (1.1) under $\left(\mathrm{f}_{2}\right)$ and the following conditions:

$\left(\mathrm{H}_{1}\right) f \in C(\Omega \times \mathbb{R}, \mathbb{R}) ; f(x, t) \geq 0$, for all $t \geq 0, x \in \Omega$ and $f(x, t) \equiv f(x, 0) \equiv 0$, for all $t \leq 0, x \in \Omega$.

$\left(\mathrm{H}_{2}\right)(f(x, t) / t)$ is nondecreasing with respect to $t \geq 0$, a.e. on $x \in \Omega$.

Note that our assumption $\left(\mathrm{f}_{1}\right)$ is weaker than $\left(\mathrm{H}_{1}\right)$. And condition $\left(\mathrm{H}_{2}\right)$ is a strong assumption.

In this paper, we prove that $\left(f_{1}\right)$ and $\left(f_{2}\right)$ are sufficient to obtain a positive solution and a negative solution of problem (1.1). Our main result is the following.

Theorem 1.1. Assume that $f$ satisfies $\left(f_{1}\right)$ and $\left(f_{2}\right)$. If $\mu<\lambda_{1}<\ell$, then problem (1.1) has at least two nontrivial solutions, one is positive, the other is negative.

Note that in Theorem 1.1, even in the resonant case, we do not need to assume any additional conditions to ensure that $J$ satisfies the (PS) condition. Thus Theorem 1.1 greatly improves previous results, such as Zhou's [12]. This fact is interesting. The proof of Theorem 1.1 will be stated in Section 2.

We can also consider the asymptotically linear Dirichlet problem for the $p$-Laplacian

$$
\begin{aligned}
-\Delta_{p} u & =f(x, u), \quad x \in \Omega, \\
u & =0, \quad x \in \partial \Omega,
\end{aligned}
$$

where $1<p<+\infty$. Let $0<\lambda_{1}^{p}<\lambda_{2}^{p} \leq \lambda_{3}^{p} \leq \cdots$ be the sequence of variational eigenvalues of the eigenvalue problem

$$
\begin{aligned}
-\Delta_{p} u & =\lambda|u|^{p-2} u, \quad x \in \Omega, \\
u & =0, \quad x \in \partial \Omega .
\end{aligned}
$$


It is known that $-\triangle_{p}$ has a smallest eigenvalue (see [5]), that is, the principle eigenvalue, $\lambda_{1}^{p}$, which is simple and has an associated eigenfunction $\varphi_{1} \in$ $W_{0}^{1, p}(\Omega) \cap C^{1}(\Omega)$ that is strictly positive in $\Omega$ and $\int_{\Omega} \varphi_{1}^{p}=1 . \lambda_{1}^{p}$ is defined as

$$
\lambda_{1}^{p}=\min \left\{\int_{\Omega}|\nabla u|^{p}: u \in W_{0}^{1, p}(\Omega), \int_{\Omega}|u|^{p}=1\right\} .
$$

Assuming $\left(\mathrm{f}_{1}\right)$ and the following condition:

$\left(\mathrm{f}_{2}^{\prime}\right) \lim _{|t| \rightarrow 0}\left(f(x, t) /|t|^{p-2} t\right)=\mu, \lim _{|t| \rightarrow \infty}\left(f(x, t) /|t|^{p-2} t\right)=\ell$ uniformly in $x \in \Omega$,

we obtain the following theorem.

Theorem 1.2. Assume that $f$ satisfies $\left(f_{1}\right)$ and $\left(f_{2}^{\prime}\right)$. If $\mu<\lambda_{1}^{p}<\ell$, then problem (1.4) has at least two nontrivial solutions, one is positive, the other is negative.

Remark 1.3. (1) The existence of a positive solution of problem (1.4) was obtained by Li and Zhou [7, Theorem 1.1], under $\left(\mathrm{H}_{1}\right),\left(\mathrm{f}_{2}^{\prime}\right)$ with $\mu=0$ and

$\left(\mathrm{H}_{2}^{\prime}\right)\left(f(x, t) / t^{p-1}\right)$ is nondecreasing in $t>0$, for $x \in \Omega$.

Condition $\left(\mathrm{H}_{2}\right)$ is a strong assumption. Moreover, if $\ell$ is an eigenvalue of (1.5), they need another condition

(f F) $\lim _{t \rightarrow \infty}\{f(x, t) t-p F(x, t)\}=+\infty$ uniformly a.e. $x \in \Omega$

to produce a positive solution. Thus Theorem 1.2 extends [7, Theorem 1.1] greatly.

(2) Obviously, Theorem 1.1 is a special case of Theorem 1.2. But we would rather state the proof of Theorem 1.1 separately, because the proof is very simple and clear.

\section{Proof of Theorem 1.1}

In this section, we will always assume that $\left(f_{1}\right)$ and $\left(f_{2}\right)$ hold and give the proof of Theorem 1.1.

Consider the following problem:

$$
\begin{aligned}
-\Delta u & =f_{+}(x, u), \quad x \in \Omega, \\
u & =0, \quad x \in \partial \Omega,
\end{aligned}
$$

where

$$
f_{+}(x, t)= \begin{cases}f(x, t), & t \geq 0 \\ 0, & t \leq 0\end{cases}
$$

Define a functional $J_{+}: H_{0}^{1} \rightarrow \mathbb{R}$,

$$
J_{+}(u)=\frac{1}{2} \int_{\Omega}|\nabla u|^{2} d x-\int_{\Omega} F_{+}(x, u) d x,
$$

where $F_{+}(x, t)=\int_{0}^{t} f_{+}(x, s) d s$. We know $J_{+} \in C^{1}\left(H_{0}^{1}, \mathbb{R}\right)$. 
512 On an asymptotically linear elliptic Dirichlet problem

Lemma 2.1. I satisfies the (PS) condition.

Proof. Let $\left\{u_{n}\right\} \subset H_{0}^{1}$ be a sequence such that

$$
\left|J_{+}\left(u_{n}\right)\right| \leq c, \quad J_{+}^{\prime}\left(u_{n}\right) \longrightarrow 0 .
$$

It is easy to see that

$$
\left|f_{+}(x, u) u\right| \leq C\left(1+|u|^{2}\right) \text {. }
$$

Now (2.4) implies that for all $\phi \in H_{0}^{1}$

$$
\int_{\Omega}\left(\nabla u_{n} \nabla \phi-f_{+}\left(x, u_{n}\right) \phi\right) d x \longrightarrow 0
$$

Set $\phi=u_{n}$, we have

$$
\begin{aligned}
\left\|u_{n}\right\|^{2} & =\int_{\Omega} f_{+}\left(x, u_{n}\right) u_{n} d x+\left\langle J_{+}^{\prime}\left(u_{n}\right), u_{n}\right\rangle \\
& \leq \int_{\Omega} f_{+}\left(x, u_{n}\right) u_{n} d x+o(1)\left\|u_{n}\right\| \\
& \leq C+C\left\|u_{n}\right\|_{2}^{2}+o(1)\left\|u_{n}\right\|,
\end{aligned}
$$

where $\|\cdot\|_{2}$ is the standard norm in $L^{2}:=L^{2}(\Omega)$. We claim that $\left\|u_{n}\right\|_{2}$ is bounded. For otherwise, we may assume that $\left\|u_{n}\right\|_{2} \rightarrow+\infty$. Let $v_{n}=u_{n} /\left\|u_{n}\right\|_{2}$, then $\left\|v_{n}\right\|_{2}=1$. Moreover, from (2.7) we have

$$
\left\|v_{n}\right\|^{2} \leq o(1)+C+\frac{o(1)}{\left\|u_{n}\right\|_{2}} \cdot \frac{\left\|u_{n}\right\|}{\left\|u_{n}\right\|_{2}}=o(1)+C+o(1)\left\|v_{n}\right\| .
$$

That is, $\left\|v_{n}\right\|$ is bounded. So, up to a subsequence, we have

$$
v_{n} \rightarrow v \text { in } H_{0}^{1}, \quad v_{n} \longrightarrow v \text { in } L^{2}, \quad \text { for some } v \text { with }\|v\|_{2}=1 .
$$

From (2.6) it follows that

$$
\int_{\Omega}\left(\nabla v \nabla \phi-\ell v^{+} \phi\right) d x=0, \quad \forall \phi \in H_{0}^{1}
$$

where $v^{+}=\max \{0, v\}$. From this and the regularity theory we have

$$
\begin{aligned}
-\Delta v & =\ell v^{+}, \quad x \in \Omega, \\
v & =0, \quad x \in \partial \Omega .
\end{aligned}
$$

The maximum principle implies that $v=v^{+} \geq 0$. But $\ell>\lambda_{1}$ and hence $v \equiv 0$ which contradicts with $\|v\|_{2}=1$. 
Since $\left\|u_{n}\right\|_{2}$ is bounded, from (2.7) we get the boundness of $\left\|u_{n}\right\|$. A standard argument shows that $\left\{u_{n}\right\}$ has a convergent subsequence. Therefore, $J_{+}$satisfies the (PS) condition.

Lemma 2.2. Let $\phi_{1}>0$ be a $\lambda_{1}$-eigenfunction of $\left(-\Delta, H_{0}^{1}\right)$ with $\left\|\phi_{1}\right\|=1$, if $\mu<$ $\lambda_{1}<\ell$, then

(a) there exist $\rho, \beta>0$ such that $J_{+}(u) \geq \beta$ for all $u \in H_{0}^{1}$ with $\|u\|=\rho$;

(b) $J_{+}\left(t \phi_{1}\right) \rightarrow-\infty$ as $t \rightarrow+\infty$.

Proof. See the proof of [12, Lemma 2.5].

Now, we are in a position to state the proof of Theorem 1.1.

Proof of Theorem 1.1. By Lemmas 2.1, 2.2, and the Mountain Pass Theorem [9, Theorem 2.2], the functional $J_{+}$has a critical point $u_{+}$with $J_{+}\left(u_{+}\right) \geq \beta$. But $J_{+}(0)=0$, that is, $u_{+} \neq 0$. Then $u_{+}$is a nontrivial solution of $(2.1)$. From the strong maximum principle, $u_{+}>0$. Hence $u_{+}$is also a positive solution of (1.1).

Similarly, we obtain a negative solution $u_{-}$of (1.1).

The proof is completed.

Remark 2.3. If we assume further that $f \in C^{1}(\Omega \times \mathbb{R}, \mathbb{R})$ and $\ell$ is not an eigenvalue of $\left(-\Delta, H_{0}^{1}\right)$, that is, $\ell \in\left(\lambda_{i}, \lambda_{i+1}\right)$ for some $i \geq 2$. Then the functional $J$ defined in Section 1 satisfies the (PS) condition. Using Morse theory, we can prove that problem (1.1) has one more nontrivial solution $u$ with $C_{i}(J, u) \neq 0$, where $C_{i}(J, u)$ is the ith critical group of $J$ at $u$.

Remark 2.4. If we assume that $\mu=\mu(x), \ell=\ell(x)$, and $\mu(x)<\lambda_{1}, \ell(x) \in L^{\infty}(\Omega)$, $\ell(x) \geq \lambda_{1}$, mes $\left\{x \in \Omega: \ell(x)>\lambda_{1}\right\}>0$, then the conclusion of Theorem 1.1 is valid too. Since under this assumption, by (2.11) we can get $\lambda_{1} \int_{\Omega} v \phi_{1}=$ $\int_{\Omega} \nabla v \nabla \phi_{1}=\int_{\Omega} \ell(x) v \phi_{1}$, thus $v \equiv 0$.

\section{Proof of Theorem 1.2 and final remarks}

In this section, we sketch the proof of Theorem 1.2 and give some remarks. First, we recall the concept Fučik spectrum and a related result.

The Fucik spectrum of $p$-Laplacian with Dirichlet boundary condition is defined as the set $\Sigma_{p}$ of those $(a, d) \in \mathbb{R}^{2}$ such that

$$
\begin{gathered}
-\Delta_{p} u=a\left(u_{+}\right)^{p-1}-d\left(u_{-}\right)^{p-1}, \quad x \in \Omega, \\
u=0, \quad x \in \partial \Omega,
\end{gathered}
$$

has a nontrivial solution, where $u^{+}=\max \{u, 0\}$ and $u^{-}=\max \{-u, 0\}$. By [5], we know that if $(a, d) \in \Sigma_{p}$ and $(a, d) \notin \mathbb{R} \times \lambda_{1}^{p},(a, d) \notin \lambda_{1}^{p} \times \mathbb{R}$, then $a>\lambda_{1}^{p}$, $d>\lambda_{1}^{p}$. We will also need the following lemma, which is due to Zhang and $\mathrm{Li}$ [11, Lemma 3]. 
514 On an asymptotically linear elliptic Dirichlet problem

Lemma 3.1. Assume that $h \in C(\Omega \times \mathbb{R}, \mathbb{R})$,

$$
\lim _{t \rightarrow+\infty} \frac{h(t)}{|t|^{p-2} t}=a, \quad \lim _{t \rightarrow-\infty} \frac{h(t)}{|t|^{p-2} t}=d
$$

If $(a, d) \notin \Sigma_{p}$, then the functional $\varphi: W_{0}^{1, p}(\Omega) \rightarrow \mathbb{R}$,

$$
\varphi(u)=\frac{1}{p} \int_{\Omega}|\nabla u|^{p} d x-\int_{\Omega} H(u) d x
$$

satisfies the (PS) condition, where $H(u)=\int_{0}^{u} h(t) d t$.

Sketch of the proof of Theorem 1.2. As in Section 2, consider the trancated problem

$$
\begin{aligned}
-\Delta_{p} u & =f_{+}(x, u), \quad x \in \Omega, \\
u & =0, \quad x \in \partial \Omega,
\end{aligned}
$$

where $f_{+}$is defined as in (2.2). Due to the maximum principle (see [10]), solutions of (3.4) are positive, thus are solutions of (1.4). We have

$$
\lim _{t \rightarrow-\infty} \frac{f_{+}(x, t)}{|t|^{p-2} t}=0, \quad \lim _{t \rightarrow+\infty} \frac{f_{+}(x, t)}{|t|^{p-2} t}=\ell
$$

Since $\ell>\lambda_{1}^{p}$, one deduces directly from the definition of Fučik spectrum that $(\ell, 0) \notin \Sigma_{p}$, thus by Lemma 3.1, we deduce that the $C^{1}$-functional

$$
J_{+}(u)=\frac{1}{p} \int_{\Omega}|\nabla u|^{p} d x-\int_{\Omega} F_{+}(x, u) d x
$$

satisfies the (PS) condition on the Sobolev space $W_{0}^{1, p}(\Omega)$ with norm

$$
\|u\|_{1, p}=\left(\int_{\Omega}|\nabla u|^{p} d x\right)^{1 / p}
$$

where $F_{+}(x, t)=\int_{0}^{t} f_{+}(x, s) d s$.

As [7, Lemma 2.3], the functional $J_{+}$admits the "Mountain Pass Geometry." Thus $J_{+}$has a nonzero critical point, which is a nontrivial solution of (3.4). From the strong maximum principle (see [10]), it is also a positive solution of (1.4).

Similarly, we obtain a negative solution of (1.4). 
Remark 3.2. Problems (1.1) and (1.4) can be resonant at infinity, this is the main difficulty in verifying the (PS) condition. But after trancating, the problems are not resonant with respect to the Fučik spectrum. Thus, from the Fučik spectrum point of view, the corresponding functionals of the trancated problems satisfies the (PS) condition naturally. And our limit conditions at zero allow us to use the trancation technique and apply the Mountain Pass Theorem.

These are the main ingredient of this work.

Remark 3.3. In fact, let $P:=\left\{u \in H_{0}^{1}: u(x) \geq 0\right.$, a.e. $\}$, the functional $J$ does not satisfies the (PS) condition on the whole space $H_{0}^{1}$ whenever $\ell=\lambda_{i}, i>1$, but from our proof $J$ satisfies the (PS) condition on $P$. That is, the unbounded (PS) sequences do not belong to $P$. This idea may be used to weaken the compact conditions for other problems.

\section{Acknowledgment}

This work was supported by the National Natural Science Foundation of China (NSFC).

\section{References}

[1] H. Amann and E. Zehnder, Nontrivial solutions for a class of nonresonance problems and applications to nonlinear differential equations, Ann. Scuola Norm. Sup. Pisa Cl. Sci. (4) 7 (1980), no. 4, 539-603.

[2] T. Bartsch and S. Li, Critical point theory for asymptotically quadratic functionals and applications to problems with resonance, Nonlinear Anal. 28 (1997), no. 3, 419441.

[3] K. C. Chang, Solutions of asymptotically linear operator equations via Morse theory, Comm. Pure Appl. Math. 34 (1981), no. 5, 693-712.

[4] Infinite-Dimensional Morse Theory and Multiple Solution Problems, Progress in Nonlinear Differential Equations and Their Applications, vol. 6, Birkhäuser Boston, Massachusetts, 1993.

[5] M. Cuesta, D. de Figueiredo, and J.-P. Gossez, The beginning of the Fucik spectrum for the p-Laplacian, J. Differential Equations 159 (1999), no. 1, 212-238.

[6] E. N. Dancer and Z. Zhang, Fučik spectrum, sign-changing, and multiple solutions for semilinear elliptic boundary value problems with resonance at infinity, J. Math. Anal. Appl. 250 (2000), no. 2, 449-464.

[7] G. Li and H.-S. Zhou, Asymptotically linear Dirichlet problem for the p-Laplacian, Nonlinear Anal. 43 (2001), no. 8, Ser. A: Theory Methods, 1043-1055.

[8] S. Li and Z. Zhang, Fucik spectrum, sign-changing and multiple solutions for semilinear elliptic boundary value problems with jumping nonlinearities at zero and infinity, Sci. China Ser. A 44 (2001), no. 7, 856-866.

[9] P. H. Rabinowitz, Minimax Methods in Critical Point Theory with Applications to Differential Equations, CBMS Regional Conference Series in Mathematics, vol. 65, American Mathematical Society, Rhode Island, 1986.

[10] J. L. Vázquez, A strong maximum principle for some quasilinear elliptic equations, Appl. Math. Optim. 12 (1984), no. 3, 191-202. 
516 On an asymptotically linear elliptic Dirichlet problem

[11] Z. Zhang and S. Li, On sign-changing and multiple solutions of p-Laplacian, preprint, 2001.

[12] H.-S. Zhou, Existence of asymptotically linear Dirichlet problem, Nonlinear Anal. 44 (2001), no. 7, Ser. A: Theory Methods, 909-918.

Zhitao Zhang: Institute of Mathematics, Academy of Mathematics and Systems Sciences, Academia Sinica, Beijing 100080, China

E-mail address: zzt@math03.math.ac.cn

Shujie Li: Institute of Mathematics, Academy of Mathematics and Systems Sciences, Academia Sinica, Beijing 100080, China

E-mail address: lisj@math03.math.ac.cn

Shibo Liv: Institute of Mathematics, Academy of Mathematics and Systems Sciences, Academia Sinica, Beijing 100080, China

E-mail address: liusb@amss.ac.cn

Weijie Feng: Institute of Mathematics, Academy of Mathematics and Systems Sciences, Academia Sinica, Beijing 100080, China 


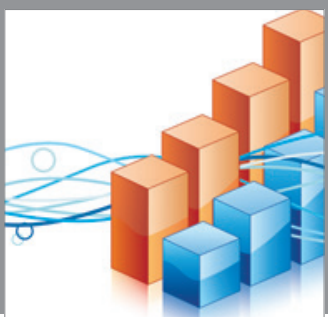

Advances in

Operations Research

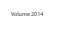

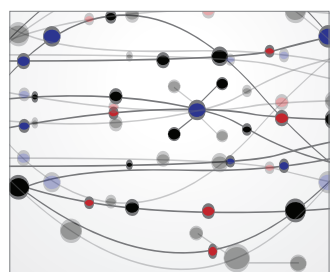

\section{The Scientific} World Journal
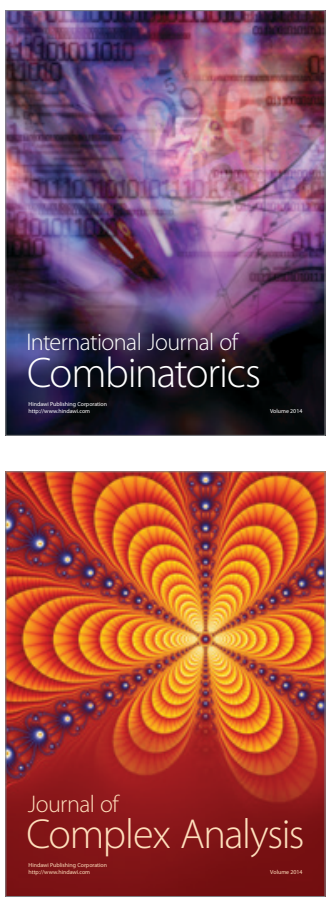

International Journal of

Mathematics and

Mathematical

Sciences
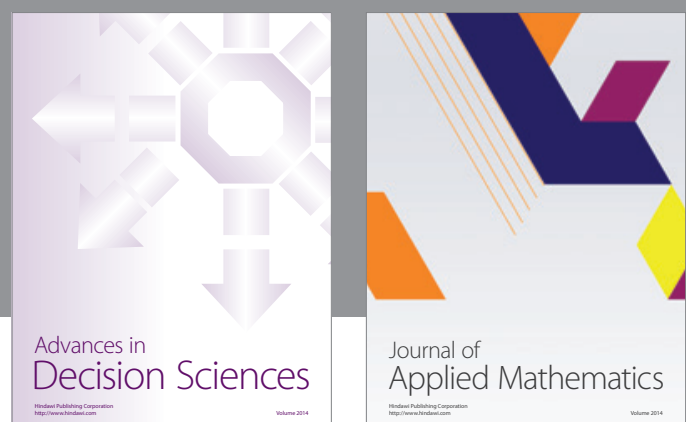

Journal of

Applied Mathematics
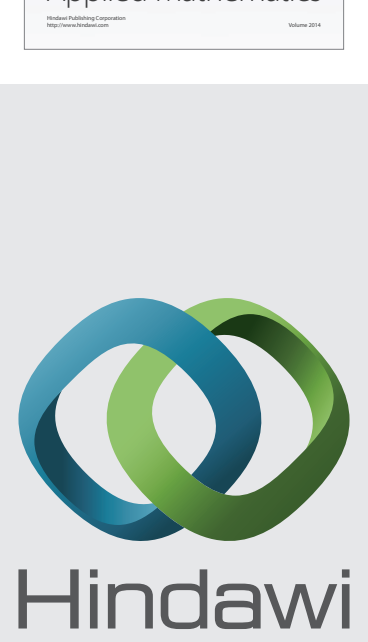

Submit your manuscripts at http://www.hindawi.com
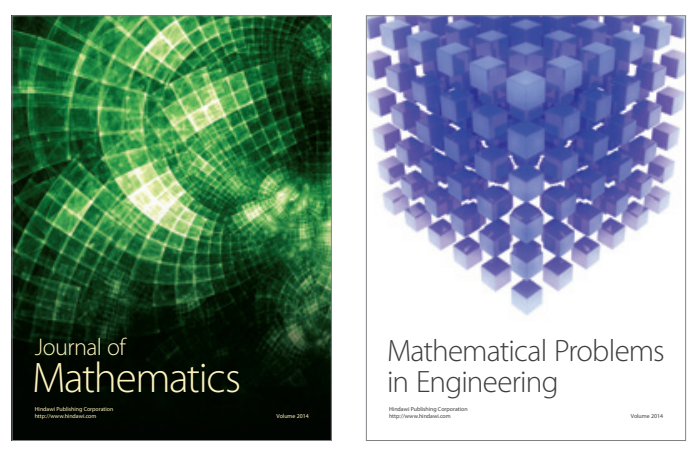

Mathematical Problems in Engineering
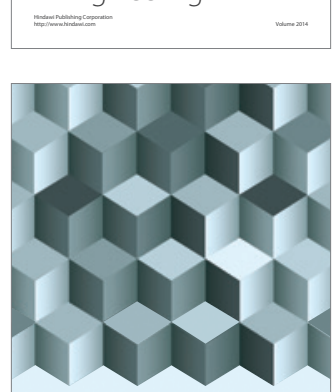

Journal of

Function Spaces
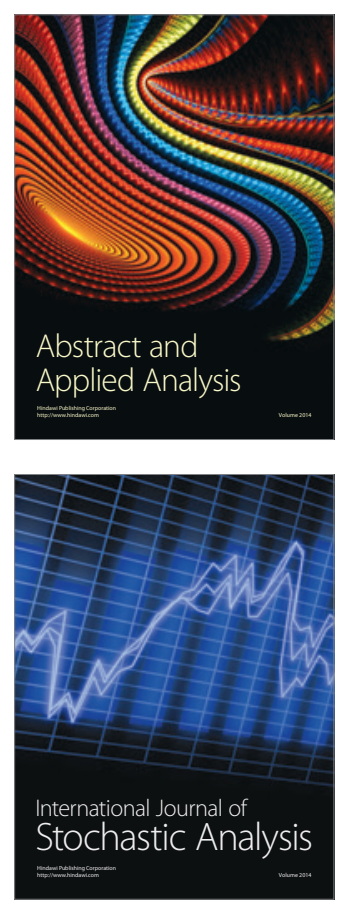

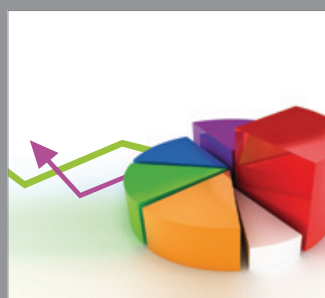

ournal of

Probability and Statistics

Promensencen
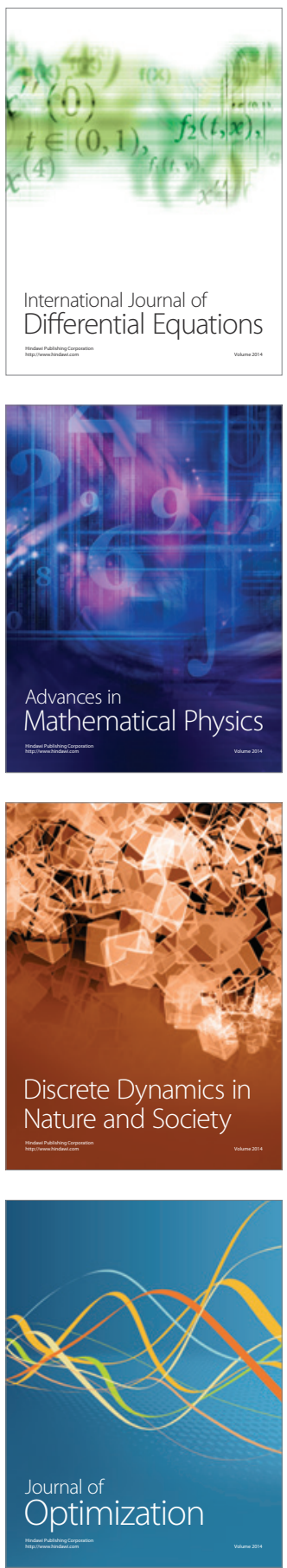